\title{
Engaging multisector stakeholders to identify priorities for global health innovation, change and research: an engagement methodology and application to prosthetics service delivery in Cambodia.
}

Cheryl D. Metcalf ${ }^{1,2}$ *

Chantel Ostler 1,2,3

Phearsa Thor ${ }^{4,5}$

Sisary Kheng ${ }^{4,5}$

Samedy Srors ${ }^{4,5}$

Ratana Sann ${ }^{6,7}$

Peter Worsley ${ }^{1}$

Lucy Gates $^{1}$

Maggie Donovan-Hall ${ }^{1,2}$

Carson Harte ${ }^{2,5}$

Alex Dickinson ${ }^{2,8}$

${ }^{1}$ School of Health Sciences, University of Southampton, UK

${ }^{2}$ Exceed Research Network, Lisburn, Northern Ireland, UK

${ }^{3}$ Portsmouth Hospitals University NHS Trust, Portsmouth NHS, UK

${ }^{4}$ Department of Prosthetics \& Orthotics, Phnom Penh, Cambodia

${ }^{5}$ Exceed Worldwide, Lisburn, Northern Ireland

${ }^{6}$ National Institute of Social Affairs, Phnom Penh, Cambodia

${ }^{7}$ National Elderly Care Center, Elderly Welfare Department, Ministry of Social Affairs, Veterans and Youth Rehabilitation, Phnom Penh, Cambodia

${ }^{8}$ School of Engineering, University of Southampton, UK

* Room 4003, Building 67, People Powered Prosthetics Research Group, School of Health Sciences, University of Southampton, SO17 1BJ, UK

Keywords: Stakeholders, engagement, international development, prosthetic, service delivery, sustainability 


\section{Abstract:}

A novel engagement methodology is presented for working with multi-sector stakeholders. The proposed methodology can be used to determine barriers and facilitators to translational research within a country-specific context by working with stakeholders in a responsive and collaborative way. A values-based stakeholder ethos throughout a research project lifecycle. This approach ensures the suitability and sustainability of research programmes being implemented within a complex ecosystem. A theoretical basis, drawn from academic and business innovation sectors, has been consolidated and adapted for practical application to design, direct and inform research in low resource settings; a complete engagement methodology is defined.

The benefit of advocating an holistic approach and embedding stakeholder engagement has been applied in the prosthetics and orthotics sector in Cambodia. Considering these issues affects the legacy of any proposed change from a research programme, supporting capacity building, sector influence and ensuring relevance to partners; promoting trust for future collaborations.

\section{Background}

Involving stakeholders as informed partners and advisors is important to make research meaningful and relevant, and translation achievable. While notable advocates encourage a qualitative methodological approach to provide a much-needed individual's voice in Prosthetics and Orthotics (P\&O) research (Dillon et al., 2019), there is often a need to understand multiple perspectives and capture wider stakeholder views. For example, this may involve using different approaches to involve a wide range of stakeholders, such as sector leaders, policy makers, influencing organisations to frame research priorities, objectives and provide insights on the reality of implementing change within the healthcare and social sectors.

Stakeholder engagement is important for health system change. Stakeholders are defined as individuals or groups who can affect or are affected by a change, and have a reciprocal, influencing relationship with those proposing change (Freeman, Harrison and Zyglidopoulos, 2018). They can influence the change but also be influenced by it. Internationally, within the Assistive Technology and Medical Devices sector, technologies have been developed and launched onto the market without engagement from the clinical community, the people who use them or the wider stakeholders who would ensure they are integrated throughout appropriate service and care models (AHRC, 2016). Aside from being an unnecessary drain on resources (time and financial) for any profit or non-profit organisation, this expedited approach to innovation and change is short-sighted can lead to poor sector engagement and products/services that are not fit for purpose. Within the broader context of health systems, successful change cannot happen without developed and sustained relationships with key stakeholders at every level of an organisation and the wider national level within health policy and governance. Not only do stakeholders often hold influence, depending on their role they may also be invested in the success or failure of any proposed change based on personal and/or professional factors. For any research, new product or service to be impactful and successful, these influencing landscapes must be appreciated, understood, and the stakeholders involved from the onset.

While co-creation and co-design are well-established fields (Sanders and Stappers, 2008; Jones et al., 2021), this paper presents a start-to-finish methodology with appropriate tools to visualise and analyse results for application within the health service design and health system strengthening domains. The methodology has evolved from well-documented value-led business modelling approaches (Osterwalder et al., 2014) and is adapted for use in health innovation and technology design. Where context is essential, this project emerged from a research partnership with an international non-government organisation (Exceed Worldwide) and a desire to improve access to prosthetic service provision in Cambodia focusing on quality, sustainability, and patient experience (Dickinson et al., 2019).

The aims of this paper are therefore addressed in two parts. Part 1 presents a conceptual framework and accompanying set of tools to guide developing, undertaking, analysing, and visualising of 
stakeholder engagement activities in global health innovation. Part 2 provides an exemplar application of the framework, highlighting stakeholder perspectives on barriers and opportunities for change in the real-world example of prosthetics service delivery in Cambodia. This two-part process contextualises the methodology and provides an opportunity to highlight important lessons from experience.

\section{Part 1 - Conceptual Framework and Definition of Tools}

The following section outlines a proposed five-phase framework of stakeholder engagement for global health innovation contexts:

- Phase 1: Revealing a Stakeholder Ecosystem: Identifying stakeholders and mapping

- Phase 2: Synthesising Stakeholder interest and influence

- Phase 3: Engaging a Stakeholder Ecosystem: Stakeholder discussions

- Phase 4: Visualising commonality: Stakeholder Value Segments and Barriers and Opportunities Motif Map

- Phase 5: Reporting: Accessible feedback to stakeholders

For each phase, an associated methodology will be defined, and visualisation tools proposed.

Phase 1: Revealing a Stakeholder Ecosystem: Identifying stakeholders and mapping

Phase 1 can be initiated as early as the development of the research funding application with partner stakeholders. It relies on initial partnerships between organisations, where one or more have knowledge and wider contacts relative to the healthcare ecosystem.

Stakeholders can be identified via:

- Exploring the roles of key organisations with initial partner stakeholders.

- Desk research to explore macroeconomics, influencing and related organisations.

- Snowballing through engagement with these different stakeholders

To produce an effective Stakeholder Map, stakeholder groups are defined at various interacting points within the project. For example, groups include patients or persons with disability (PWD), patient advocate groups, healthcare insurers, private hospitals, public hospitals, clinical groups, nongovernment organisations, government organisations or industrial sector organisations, and others depending on the context. Error! Reference source not found. provides a simplified illustration of a Stakeholder Map adapted from Freeman (1984). Stakeholder maps can take the form of a visual map as depicted by Freeman (1984), or in the form of a table (Mtika, Wilcox and Feune De Colombi, 2021). 


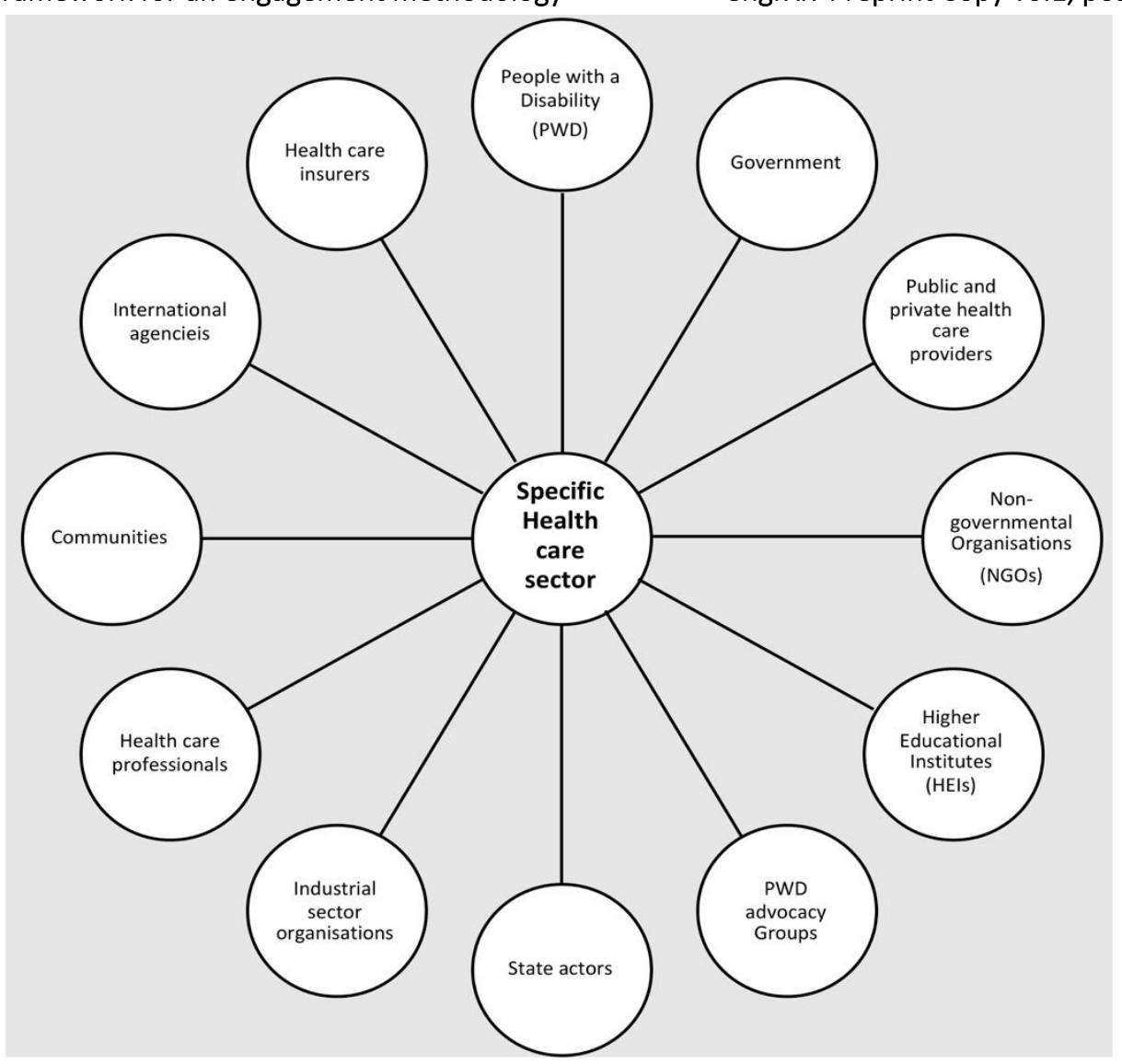

Figure 1: Example Stakeholder Map showing a representation of stakeholders (actors) within a health sector in a Low Resource Setting. Adapted from Freeman (1984).

\section{Phase 2: Synthesising Stakeholder interest and influence}

Stakeholder segments, or groups of stakeholders that have similar influences, can be classified into comparator groups, for example all manufacturers, all hospitals in the private sector, all hospitals in the public sector, or all international or bilateral donors. These can then be classified using a Mendelow Matrix (Johnson, Scholes and Whittington, 2008) (Figure 1) to further analyse the level of influence a stakeholder has with reference to a project, development or service. This can be done with the initial partner stakeholders as part of a workshop, but the Matrix should be seen as a flexible creation, evolving throughout the project as more stakeholders' views are incorporated. 
Level of Interest

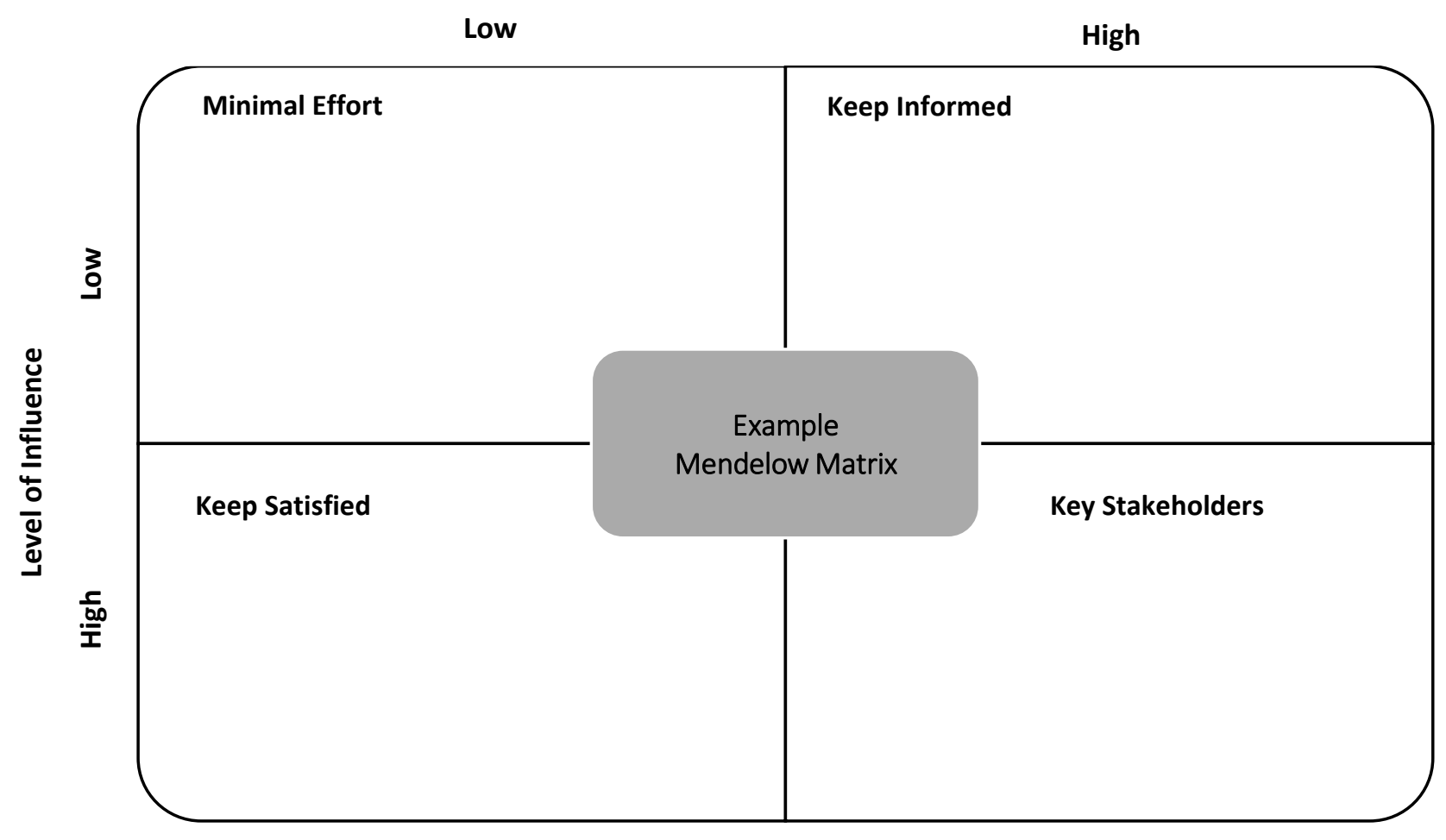

Figure 1: Example Mendelow Matrix defining the quadrants for classification of stakeholders' levels of interest and influence.

Phase 3: Engaging a Stakeholder Ecosystem: Stakeholder discussions

Initial partner stakeholders can act as facilitators to introduce and coordinate discussions with other stakeholders (e.g., government officials or policy makers/influencers). Discussion guides are developed specifically for each stakeholder group. Open, non-leading questions form the basis of these discussions, where project holders are not guiding or limiting the discussion, but where the focus is based upon a particular area of common interest. These discussions require the participation of a minimum of one member of project staff and one partner stakeholder. Within the discussion, notes are taken which then form the basis of a post-discussion reflective session within the project team and initial partner stakeholders. This reflective session provides an important opportunity to verify interpretations of the discussion, allow for sociocultural context to be included and, if necessary, allow for post-translation questions within the team.

Phase 4: Visualising commonality: Stakeholder Value Segment and Barriers and Opportunities (BaO) Motif Map

Stakeholders are grouped based on common factors, such as type and power of influence (referencing Phase 2 above). A 'motif' in this context is a recurring idea identified and consolidated for each group, and common motifs are highlighted when they emerge from more than one group. This process can be visualised using the Stakeholder Value Segment (Figure 2), which was adapted from Customer Segment Analysis (Osterwalder et al., 2014) specifically for use within this framework and the global health context. This phase is iterative and can be revisited throughout the lifecycle of a project, adding a layer of legitimacy to the motif by continuous monitoring, evaluation, and adjustment with additional stakeholder discussions. One of the main advantages of this phase is allowing the stakeholder an opportunity to propose solutions, which can then be highlighted during reporting (Phase 5). This provides the opportunity for championing of any embryonic solutions by stakeholders and is a critical component of relationship building and acceptance of change. Value is 
thereby defined by what stakeholders perceive as important, and this method allows them to see value reflected in any future proposal for change.

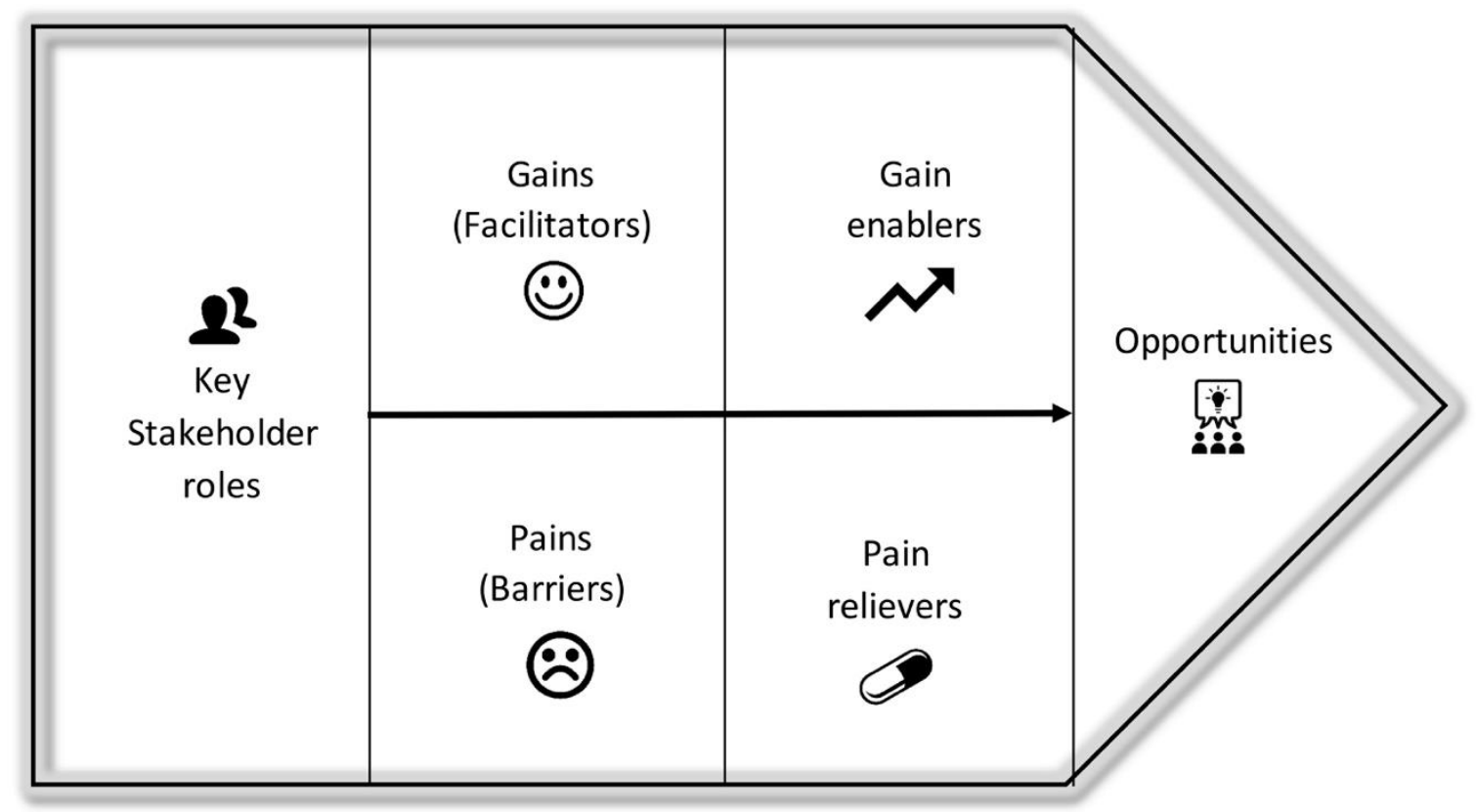

Figure 2: Stakeholder Value Segment can be used to articulate the motif emerging from a group or groups of stakeholders.

Having a method of visualising stakeholder value in the topics that were discussed requires a new method of presentation: the BaO Motif Map (Figure 3). The BaO Motif Map is divided into two types of influencing factors: 'Barriers' and 'Opportunities'. A motif is presented as a single, discrete theme where each motif depicts a barrier or opportunity identified by the stakeholder groups. Barriers are depicted on the outside and opportunities on the inside of the circle. Importantly, overlapping Barrier and Opportunity motifs (named Pivotal Motifs) highlight areas of commonality, which are important as identifiers of strategic importance in the success or failure of any proposed innovation or change. These motifs should be carefully managed as their innate complexity could influence the success or failure of any innovation.

The $\mathrm{BaO}$ Motif Map represents barriers and opportunities at a single point in time, and should any of these factors change, the map can rotate, expand, or contract. With this flexibility, the BaO Motif Map could also be used to track progress within a sector over time or be used to compare stakeholder views across related sectors (health and social care, for example) or countries. The outcomes elucidated from the $\mathrm{BaO}$ Motif Map can be included in further evidence alongside the macroeconomic environment and context analyses such as STEEPLE (More, Probert and Phaal, 2015)/PEST [LED] (Whittington et al., 2020), Lewin's Force Field Analysis (Lewin, 1951) and Porter's Five Forces (Porter, 1979). 


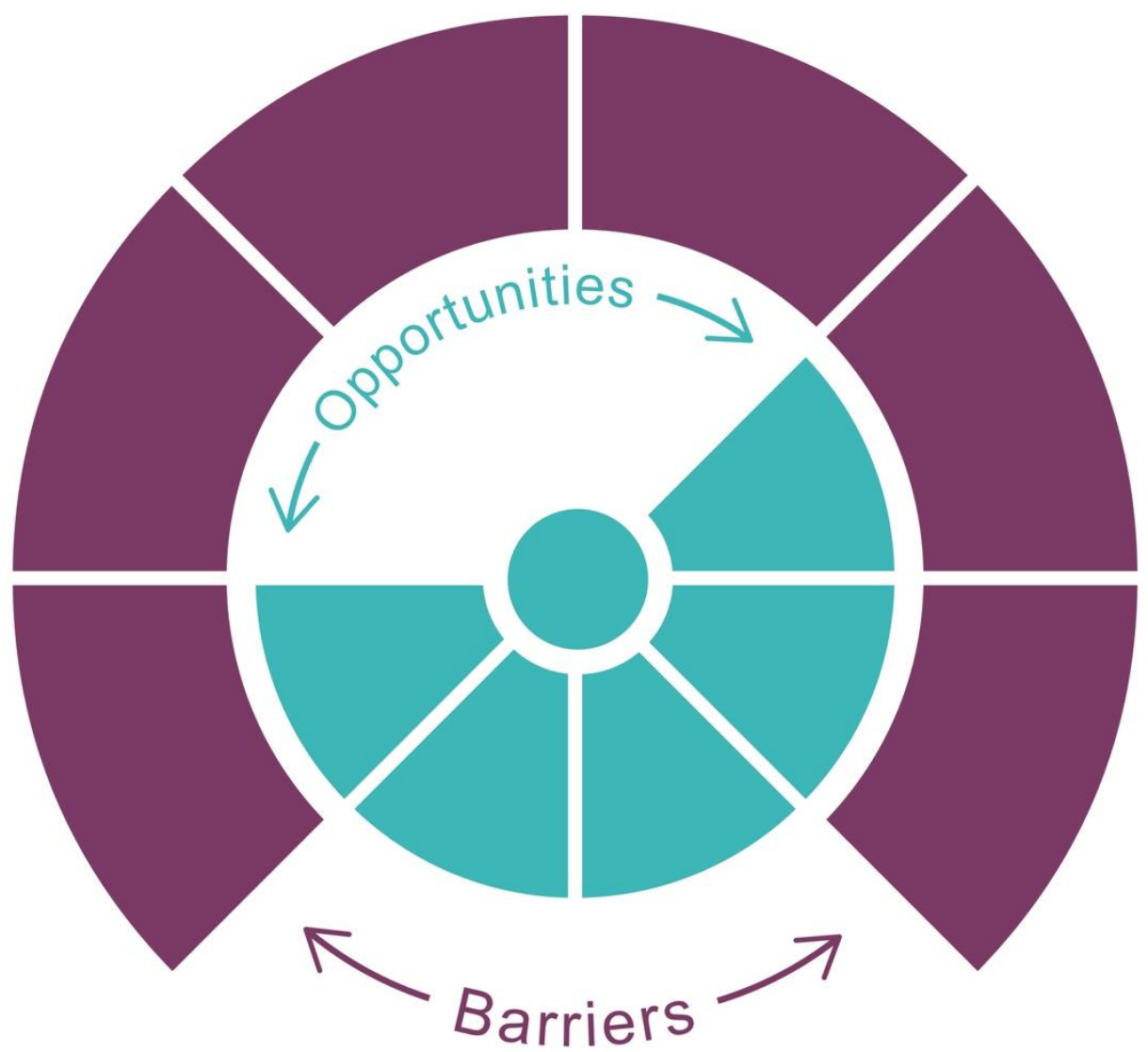

Figure 3: The Barriers and Opportunities (BaO) Motif Map, which visualises discrete motifs, identifying them as a Barrier or Opportunity, and allows clear visualisation of Pivotal Motifs that are both a Barrier and an Opportunity.

Phase 5: Reporting: Accessible feedback to stakeholders

The final iteration of this partnership process is to provide a structured report back to stakeholders. This allows them to leverage the synthesised motifs to advocate for change. This is an important, and often overlooked step in establishing a reciprocal and sustained relationship with stakeholders and organisations. Depending on the stakeholder's influence, the report can be used to inform research priorities, national policy, strategy development and social change. Importantly, by using this method, all stakeholders will be able to recognise their own influence and perspective in the report, as well as those alternative perspectives, where there might be a collective opportunity to solve or leverage change aligned with motifs. Conflicting views would be articulated in Pivotal Motifs and a more granulated overview would be presented in the structured report.

\section{Part 2-Exemplar Application: A stakeholder perspective to barriers and opportunities for change in P\&O service delivery in Cambodia.}

The following section provides an exemplar application of the proposed conceptual framework and associated tools. This exemplar will illustrate the use of the proposed framework and highlight lessons learnt from a real-world application.

\section{Exemplar application background}

A relationship was developed between academic partners and our initial partner stakeholder. The focus of this collaboration was to explore and understand the barriers to and opportunities for change in P\&O service delivery in Cambodia from a stakeholder perspective.

Approximately 100 million people worldwide need P\&O devices (WHO, 2013). In low-income and lowand middle-income contexts (collectively referred to here as low resource settings - LRSs), low-cost, robust prostheses and orthoses are not the main barrier to care: an estimated 80 - $90 \%$ do not have access to $P \& O$ services 'due to a shortage of personnel, service units and health rehabilitation 
infrastructures' (Sexton, 2015). However, the benefit of providing such services was recently highlighted by identifying that $86 \%$ of people who have a prosthetic device would describe themselves as being employed (CHAI/ATScale, 2020). Therefore, the wider socioeconomic and direct patient benefits cannot be underestimated, and this provides a powerful context for innovation and change alongside global equality initiatives such as the WHO Rehab2030 (WHO, 2019) and the UN Sustainable Development Goals (United Nations, 2015) and Universal Health Coverage.

Access to P\&O services is particularly poor for people in LRSs, who are typically younger and can often have higher physical working demands than those in high income countries, for whom most P\&O technology has been developed and where services are often more readily accessible. The demographics of those affected also differs, with some LRSs having high levels of traumatic amputation from road and workplace accidents, conflict and landmine injuries, and humanitarian crises (Marino et al., 2015). By contrast, the primary reason for amputation in high income countries is vascular compromise associated with diabetes, although the prevalence - predicted to be 700 million people by 2045 - is growing fastest in LRSs (Saeedi et al., 2019).

Over the last 40 years, Cambodia's healthcare service has been heavily supported by international aid donors. However more recent humanitarian crises require these donors to refocus their effort to other countries and are therefore slowly withdrawing support in Cambodia. In recent years, bilateral government aid arrangements - for example, the Department of Foreign Affairs and Trade of Australia and the Korean Overseas International Cooperation Agency - focus increasingly on disability policy development in the country. Despite such major international contributions, significant challenges still exist facing professional service delivery to people with disabilities due to financial hardships at a grass-root level (Bailey, 2015).

The aim of this exemplar application is to demonstrate the use of the presented conceptual framework and tools and contextualise them for a use case in the Cambodian P\&O sector. Following the Patient and Public Involvement framework (NIHR, 2012), ethical approval was not sought for this exemplar as stakeholders are not participants in research; rather, they are future partners to ensure the sustainability of any research or innovation initiatives. Unlike qualitative research, the findings from this project are attributed to all stakeholders as a group, rather than individuals. Again, because stakeholders are future partners instead of research participants, it is not appropriate to present quotations.

\section{Exemplar application method}

Phase 1: Revealing a Stakeholder Ecosystem: Identifying stakeholders and mapping The project was co-developed between the University of Southampton (UoS, research team) and an international non-government organisation (INGO) Exceed Worldwide (EW, initial partner stakeholder), collectively known as the project team. EW 'supports people with disabilities living in poverty by providing free P\&O services in South and Southeast Asia' (EW, 2021). Through their work in the region, EW had observed the paucity of services and the depletion of the international aid model to financially support P\&O services in the long term. The project team conceptualised a technology-focused research project to widen access to services, however wanted to incorporate wider stakeholder views in the proposal for change. Mapping the stakeholder ecosystem was undertaken via a series of meetings with EW staff exploring pathways of care, multidisciplinary teams, governance, supporting organisations, patient groups, health care systems, wider INGO involvement and associated professional organisations. This was supplemented with a period of desk research to widen and consolidate the team's understanding of national and international influencing factors, such as the United Nation's Sustainable Development Goals and the WHO Rehab2030 agenda. This was followed by further meetings with the partner stakeholder to build upon and verify the emerging ecosystem.

Figure 5 summarises the types of stakeholders included in the map. An extensive range of stakeholders were mapped to capture actors within the field and allow the team to build knowledge about their involvement, influence, and priorities, and understand how they interact. 


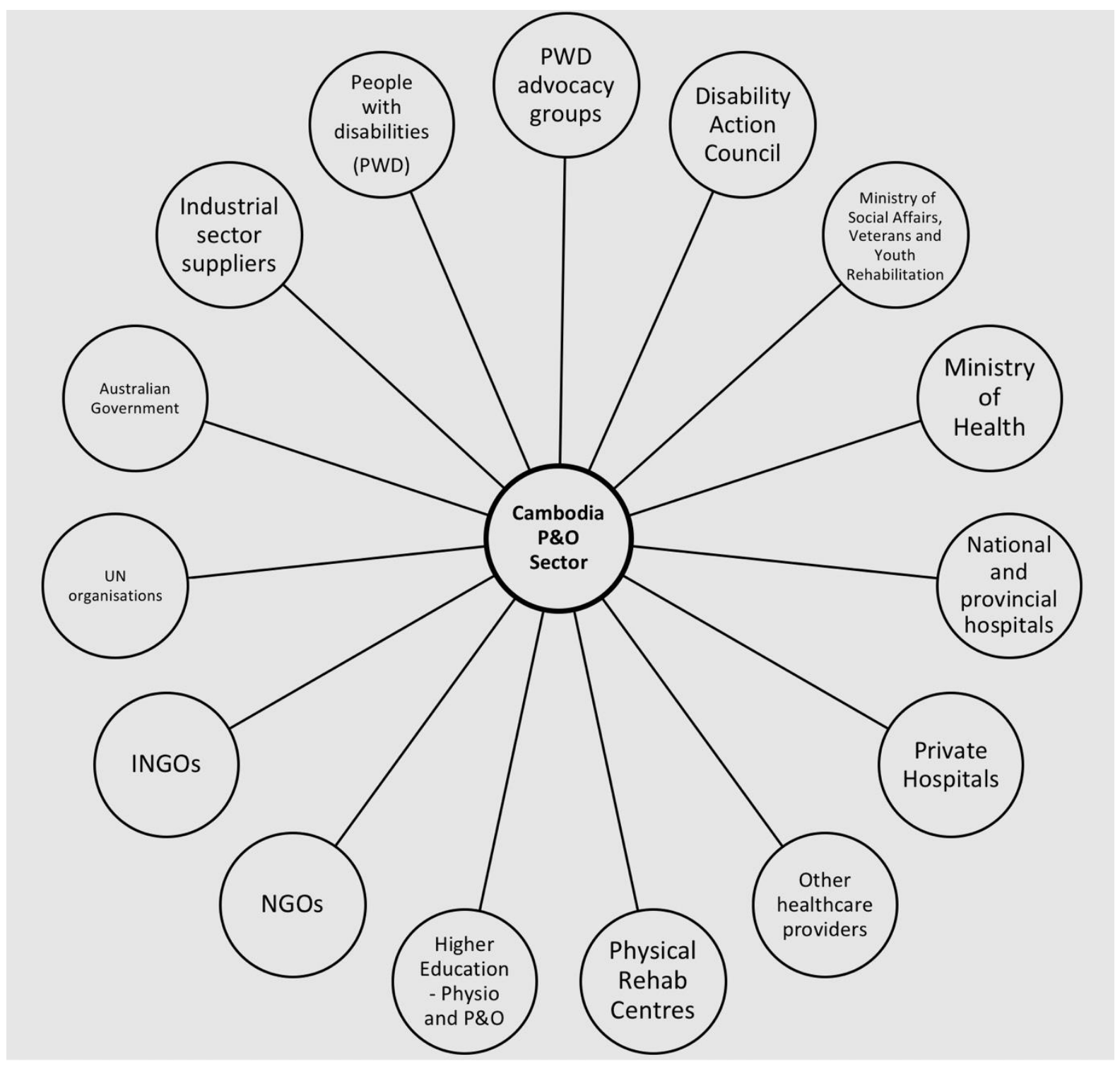

Figure 5: Summary of Cambodian Prosthetics \& Orthotics sector stakeholders identified as part of the stakeholder mapping (Phase 1). A complete version can be found in the supplementary online materials.

\section{Phase 2: Synthesising Stakeholder interest and influence}

In collaboration with EW, stakeholders were identified who had the most interest and influence, and whose views and insights would be most valuable to understand how the sector would respond to change (Figure 6). 
Level of Interest

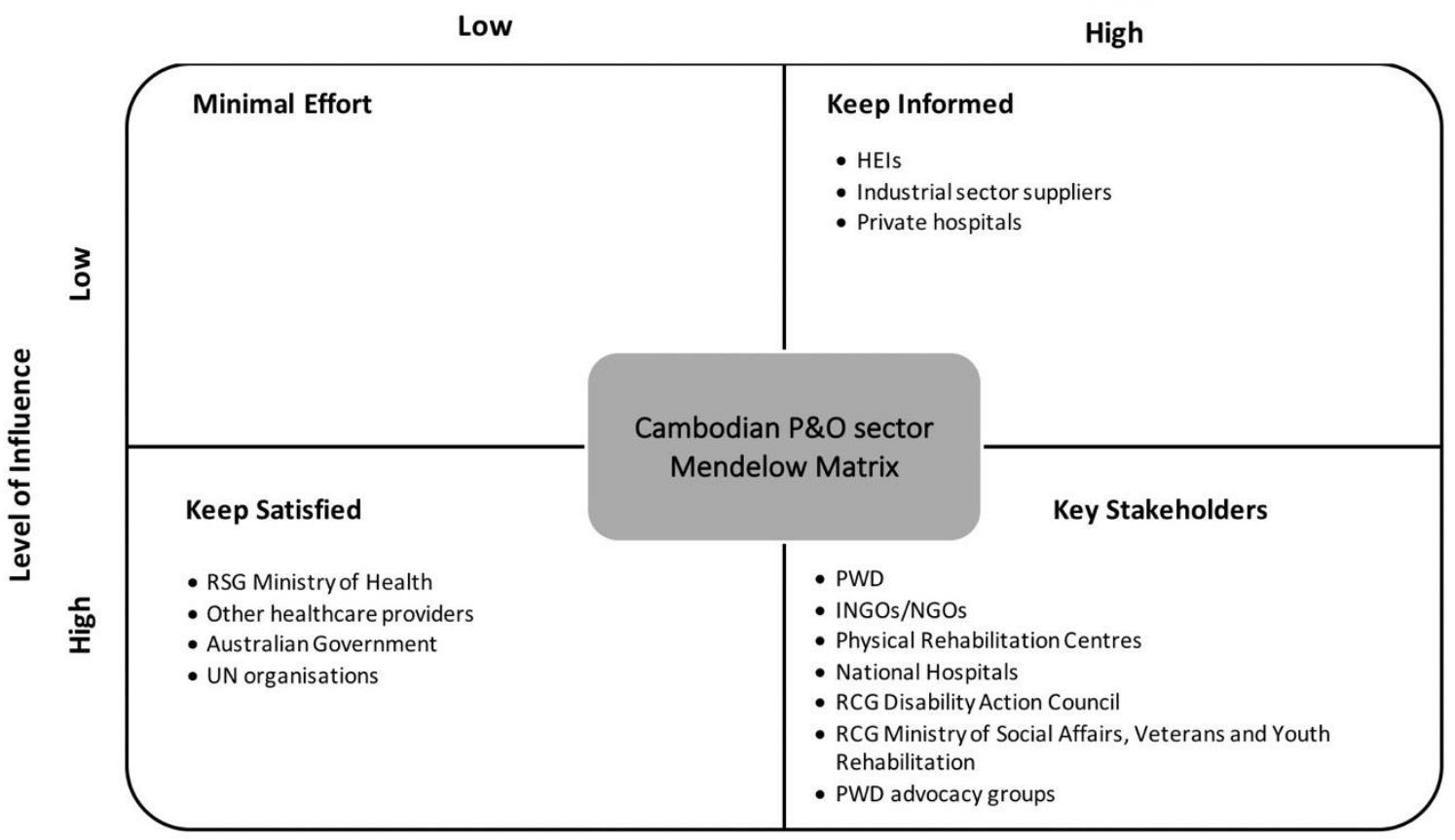

Figure 6: Cambodian prosthetics \& orthotics sector Mendelow matrix of influence vs. interest.

Phase 3: Engaging a Stakeholder Ecosystem: Stakeholder discussions

EW were crucial to engaging with the key stakeholders identified in phase 2 . They sourced well-placed stakeholders within each group and acted as advocates to introduce the project team, explain the project and co-ordinate a schedule of meetings. Discussion guides were developed for each group and based on initial partner stakeholder feedback and desk research.

Meetings often took different formats depending on who the stakeholders were, varying from discussions with prosthetic users in their homes to formal meetings with members of the Royal Cambodian Government (RCG). This also led to variation in translation practices, which reflected the needs of the stakeholder at the time. For example, two translators worked formally during government meetings, some meetings employed a single translator and others were undertaken in English. Notes were taken throughout the meetings by two members of the project team.

An important reflection during the application to Cambodia was the crucial interfacing role the initial partner stakeholder played, which appeared key to the success of this phase. The best outcomes were achieved when partner stakeholders were able to approach key stakeholders at all levels. They were pivotal to the success of the meeting arrangements. In this situation, it was also important that the initial partner stakeholder was fully engaged in the development of the overarching project, as well as the previous phases described above, so that they were able to stakeholders with context the wider project agenda.

\section{Phase 4: Visualising commonality - Barriers and Opportunities (BaO) Motif Map}

Themes were developed iteratively from amalgamated field notes. On completion of the Value Stakeholder Segment, BaO Motif Map was produced (Figure 7), which describes the P\&O sector at a single point in time (October 2019) and visualises the themes from the P\&O stakeholder engagement in Cambodia. 


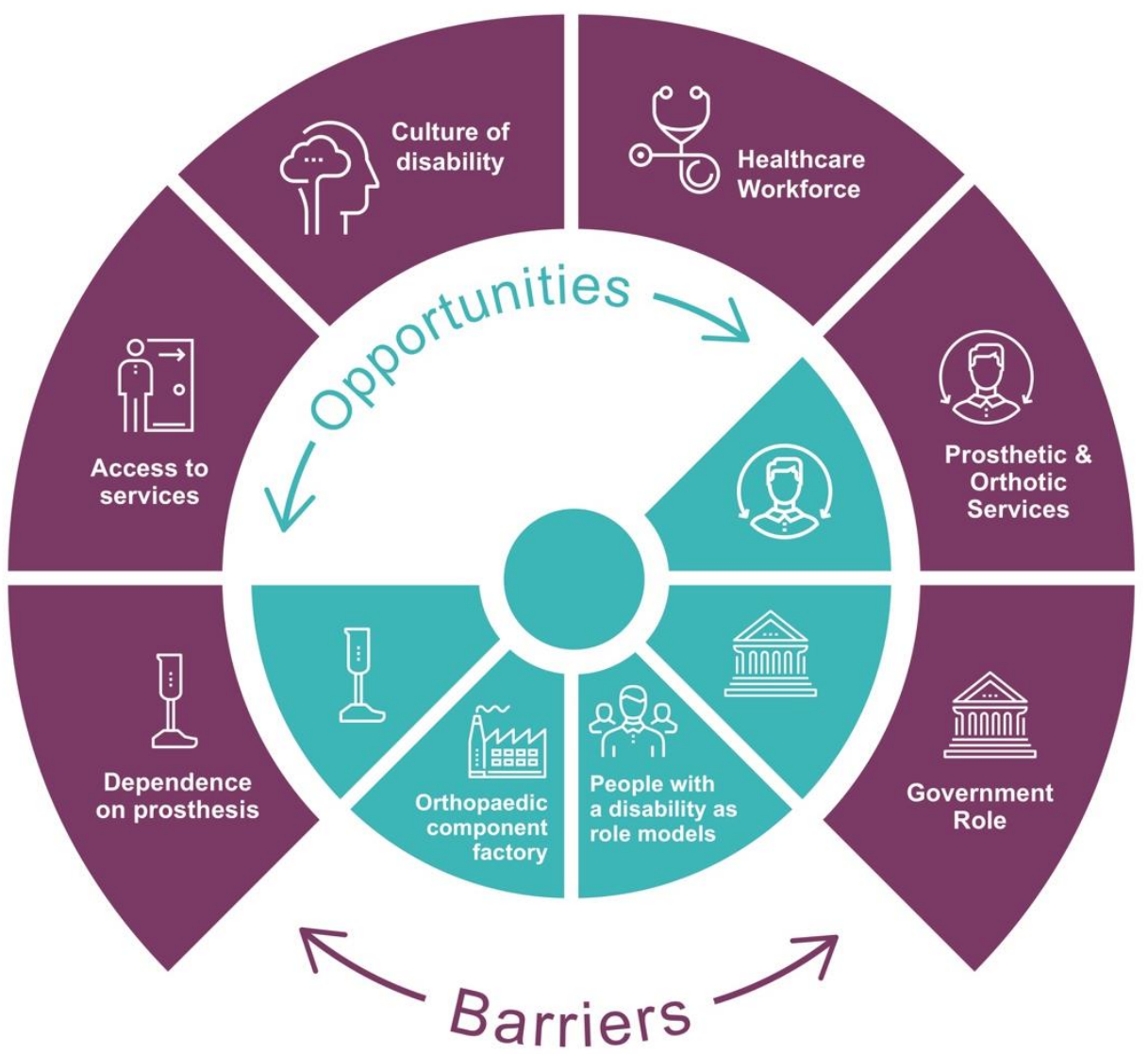

Figure 7: Barriers and Opportunities Motif Map for the P\&O sector in Cambodia, as of October 2019.

Phase 5: Reporting: Accessible feedback to stakeholders

The findings, including the $\mathrm{BaO}$ Motif Map, were summarised in a variety of formats for stakeholders. A communication plan was created to understand the key messages from the findings and their value to the different stakeholders. Appropriate and accessible methods of communication were discussed with the initial partner stakeholder EW to ensure that feedback took the right format for each stakeholder group to maximise dissemination. For example, a written report was provided to Higher Education Institutions and departments of the RCG, whereas animated videos and Facebook posts were created for clinicians and PWDs, with links available to more detailed summaries. Online seminars and symposia at an international congress were held for NGOs, service funder and academic researcher stakeholders.

\section{Barriers to change}

\section{Healthcare workforce}

Concerns were raised by several stakeholders about the availability of qualified and experienced healthcare professionals to deliver post-amputation prosthetic rehabilitation services in Cambodia. Although degree-level courses in both Physiotherapy and Prosthetics and Orthotics are available in the country, these roles are not well understood or respected by the wider public, thus impacting national workforce recruitment opportunities. Despite support in recent years from INGOs offering bursaries, uptake of training places remains poor. As well as limited numbers of new graduates, there are only small numbers of Physiotherapists and Prosthetists currently practicing. Stakeholders felt that poor awareness of the role by the public and key referrers, such as Doctors and Surgeons, can lead to underutilisation of services and patients getting lost along the post-amputation rehabilitation pathway. There is an intrinsic link to health system strengthening and integrated services with this theme. 
Low wages of P\&O and Physiotherapy staff was identified as a key driver affecting the uptake of training places and the retention of qualified staff. For Physiotherapists, this can result in a pull to the more lucrative private sector, leaving notable skill gaps within public services. Private roles are also used to supplement low wages within the public sector, but these opportunities are currently not available for clinical Prosthetists/Orthotists or Prosthetic and Orthotic Technicians. Their wages and conditions of employment are often disadvantageous as they are not awarded civil servant status. Stakeholders involved in service delivery were concerned that this gap in the current prosthetic rehabilitation workforce makes service delivery challenging both now, and in the future, and will have a notable impact on the availability, effectiveness, and quality of prosthetic care (Bailey, 2015). It is important to note that at the time the study was conducted key roles in the prosthetic multidisciplinary team (MDT), such as Occupational Therapists and Doctors of Rehabilitation Medicine were absent from prosthetic services and higher educational training in Cambodia.

\section{Access to services}

Access to health care services is a known problem throughout LRSs (Marino et al., 2015), and Cambodian P\&O services are no exception with stakeholders highlighting that access is challenging for people with both new and established amputations. Following a new amputation, many people face lengthy delays before they are fitted with a prosthesis due to an absence of widespread referral systems post-surgery and continuity of care between health and physical rehabilitation services. Stakeholders identified factors that contribute to delays in accessing services, notably the lack of awareness from potential medical referrers, and concerns over costs in a health care system with high out-of-pocket expenses. These delays may have a significant impact on the individual's physical, psychological, and socioeconomic stability and recovery.

The cost of attending a P\&O service was also thought to be prohibitive for many patients, especially those from more rural areas. The monetary impact of travelling to the centre, accommodation costs whilst limbs are manufactured and time away from family and earning an income can lead to a significant financial burden for the patient or result in non-attendance. INGOs often have financial support available for transport, meals and accommodation expenses in the form of reimbursement, however many stakeholders felt this did not resolve these issues, as money still had to be found upfront for costs such as travel to the clinic. The lifelong requirement for prosthetic repair, renewal and replacement following an amputation, raised concerns amongst stakeholders that the monetary impact of accessing P\&O services over time can lead to a spiral of poverty and disability (Cobley, 2018) as costs accumulate.

\section{Culture of disability}

Stakeholders described a culture of disability in Cambodia associated with stigmatization and made comments to the perception of previous 'bad karma' and punishment. Stakeholders described situations where individuals were socially excluded, unable to gain employment and struggled to access education following amputation. People with an amputation were often described as the poorest people in their communities and these aspects of socioeconomic status, and perhaps social stigma, were viewed as linked to poor health and poor health seeking behaviours, respectively. This is well known as the Disability Poverty Nexus or Spiral (Dalal, 2010). First line health care was frequently described as being sought from local (often unqualified) pharmacists rather than public health centres. Stakeholders felt that this perception of people with a disability as struggling for acceptance in society led to them becoming very dependent on any support available. This in turn was described as negatively affecting their independence and autonomy, which may impact their ability to raise their voice and defend their newly established rights within the eyes of the government.

\section{Both barriers and opportunities}

\section{Prosthetic and Orthotic services}

Cambodia today has a country-wide network of Physical Rehabilitation Centres, delivered by or with support from INGOs, to provide P\&O services to people following amputation. As Cambodia has strived to move away from reliance on aid toward a more sustainable, publicly provided service, the 
Persons with Disabilities Foundation (PWDF) was established within the Ministry of Social Affairs, Veterans and Youth Rehabilitation (MOSVY) to realise this ambition.

Stakeholders identified this existing network and infrastructure, with its well-established government and INGO partnerships, as an excellent platform for development. However significant variation was highlighted in service provision across the country, especially in newly transitioned public services where several stakeholders, including representatives from government, identified that public services lack the budget and knowledge to provide the required standard of care compared to INGOs (Bailey, 2015).

Stakeholders highlighted that variation across services is compounded by a lack of focus within the sector on quality-of-service provision, efficiency, and outcome. Stakeholders reported that due to the above-described workforce issues, MDT working is a challenge for most centres and many focus on the provision of prosthetic devices rather than providing rehabilitation. Stakeholders felt that improved leadership and co-ordination and transparency across the sector would promote greater partnership working at a national health systems level and more equitable service provision.

\section{Government Role}

The World Health Organisation Rehab2030 strategy (WHO, 2019) highlights the need for rehabilitation services to be aligned with wider health care systems and be governed by a country's Ministry of Health to ensure rehabilitation is included in efforts towards universal health coverage. Cambodian P\&O services sit within the remit of the Ministry of Social Affairs, Veterans and Youth Rehabilitation (MoSVY) rather than the Ministry of Health, and although cross ministry working is apparent, P\&O services were described by stakeholders as being viewed as a social intervention. Stakeholders in this project felt that the MoSVY struggled with a restricted budget when compared to the Ministry of Health, which resulted in low wages, lack of civil servant status for employees and selfacknowledged challenges contributing to, and ensuring, equitable access to welfare initiatives. However, despite these challenges there appeared to be a drive for collaboration from all government stakeholders involved, and the development of a new national centre is intended to bring together health and rehabilitation services, assistive technology, and manufacturing with the aim of highlighting this level of service provision both nationally and internationally.

\section{Dependence on prostheses}

Many of the stakeholder meetings were with users of prosthetic devices. They reported that their prosthesis allows them the chance to make a significant recovery, by re-establishing employment opportunities, the ability to travel, acceptance and engagement within their community. They described being very dependent on their prosthetic devices, demonstrating a significant need for service provision within the country. However, this dependency was felt to have mixed consequences, with any threat to limb wearing, such as prosthesis damage, being described as having a major impact on choices affecting their participation and way of life, for example engaging in family and community activities or maintaining employment.

\section{Opportunities to support development and capacity building}

\section{People with a disability as role models}

Stakeholders described how people with disabilities in Cambodia face challenges associated with social stigma every day, but many can find meaningful employment, participate in education, and fulfil roles within their communities. Stakeholders in this project, especially prosthesis users felt PWD need to become role models for independence and empowerment. They highlighted important opportunities to actively break the stigma of disability, provide peer support for others with a disability and advocate for people in need. Although this group is unlikely to represent the views of disabled people throughout and beyond Cambodia, it is important to recognise the need for PWD to have their voices heard and the opportunity to embody the changes they wish to see in their society. As a group they are important stakeholders to involve as they are both highly interested and influential amongst their peers and are increasingly involved at a government policy level. 
The handover of technical, financial and managerial responsibility of the Cambodian Orthopaedic Component Factory to the MoSVY from the International Committee of the Red Cross was completed in 2018. This established, and regionally connected organisation, facilitates opportunities for income generation for the MoSVY. The P\&O devices they produce are an appropriate technology, beyond simply matching rural Cambodian users' requirements of durability and low initial cost. They are also commercially and environmentally sustainable, with on-site polypropylene recovery and recycling from manufacturing waste and returned $\mathrm{P} \& \mathrm{O}$ devices reducing dependence on importing material. Development of new prosthetic products and manufacturing techniques, as well as the production of wider assistive technology to meet the social and cultural needs of Cambodian people, was viewed as presenting significant opportunities for development within the country and international export throughout the region.

\section{Overall discussion}

While qualitative research is focused and in-depth (Braun and Clarke, 2013) this paper presents a methodology for engagement with wider stakeholders to understand the breadth of perspectives that influence innovation and change, whether from a government or policy perspective, or from a clinical and service user perspective. This is not presented as a research methodology, but an engagement methodology. It is not intended to produce quantifiable results or qualitative themes, but to be used as a means of exploring and understanding the complex landscape in which development takes place, and to ensure that the research it defines as a result is meaningful, locally owned, collaborative and fit-for-purpose. The approach proposed is an intersection between market analytics within business development (Osterwalder et al., 2014) and the Public and Patient Involvement framework (INVOLVE, 2016) that involves members of the public, wider stakeholders, and users of health services as partners in research, rather than just as participants. As an outcome, it provides a collective understanding and language to facilitate productive and impactful research. Specifically, stakeholders are involved in 'prioritising research' and 'commenting on the feasibility of the research design' in the phase prior to participant research being undertaken (INVOLVE, 2016). This stakeholder-led methodology determines the value of any perspectives on proposed change resulting from research and therefore makes positive steps to ensure subsequent research is appropriate, meaningful, and has the best chance of use translation through clinical and service adoption.

The application of the methodology to a Cambodian P\&O sector exemplar highlighted areas of influence and the potential barriers and opportunities from different stakeholder perspectives, which helped to broaden our understanding of the current issues they face. The considerable impact that prosthetic provision has on the lives of people following limb loss is evident. These findings contribute to the growing case for ongoing funding and provision of services in Cambodia to both rehabilitate people with a prosthetic limb and to ensure their continued independence through effective and timely maintenance services.

As in many LRSs, providing and sustaining a skilled workforce to deliver health care services is a major challenge. The International Society for Prosthetics and Orthotics suggest that the number of P\&O professionals, as well as technicians, does not meet this recommended provision in high income countries, and in Southeast Asia the available workforce may be as low as one tenth of the number required (WHO, 2017a). The WHO estimate there are less than 10 skilled rehabilitation professionals per 1 million population in many LMICs (WHO, 2019). With an estimated $0.5 \%$ of the global population in need of a prosthetic device - equivalent to 35-40 million people (WHO, 2017b) - and the increasing global prevalence of non-communicable diseases, road traffic accidents and workbased accidents, this requirement is only going to increase.

In Cambodia training and providing a sustainable multidisciplinary workforce to deliver P\&O services is difficult. Both $\mathrm{P} \& \mathrm{O}$ and Physiotherapy training programmes struggle to fill their places, and programmes for other prosthetic multidisciplinary team roles do not yet exist within the country. 
Khan et al., (2018) suggested that specialised multidisciplinary teams, such as those seen within prosthetic rehabilitation may not be feasible in less-resourced settings, and alternative approaches should be considered such as task shifting, supporting community-based programmes and the use of telerehabilitation. However, such innovations should be undertaken in a systematic way to ensure quality and safety, and not be adopted at the expense of investment in training (WHO, 2008). Who the tasks are shifted to, particularly in rehabilitation settings, should also be carefully considered as previous studies within stroke rehabilitation in LRSs have found shifting to family members is not effective (Lindley et al., 2017; Zhou et al., 2019).

Access appears to be an important barrier to prosthetic use and services must strive for greater accessibility, particularly focusing on how best to support patients getting to services that are available (Dickinson et al., 2019). New models of care should be considered that are not based on historical High Income Country ways of working but develop out of, and in response to, the needs of local contexts, such as decentralised systems with mobile teams and transportable manufacturing solutions (CHAI/ATScale, 2020).

Service should go beyond just the provision of assistive technology, and crucially quality should also not be overlooked in the struggle to improve access (Matter et al., 2017). Variation in prosthetic providers in Cambodia inevitably leads to variation in service provision. A nationwide service specification or minimum package of activities is necessary and should strive for a user-centred rehabilitation approach (WHO, 2019). The future inclusion of quality indicators, agreed in partnership with current stakeholders and service providers, alongside an understanding of what outcomes are important to capture in Cambodia (Dickinson et al., 2019), could assist in equitably raising the quality of prosthetic rehabilitation and assist in the evaluation of services to improve efficiency, quality, and user experience.

This study has also highlighted opportunities for engaging further with key stakeholders in the development of future projects to address the findings presented here. Important stakeholders in this sector are the users of prosthetic services. People with disabilities (PWD) in Cambodia face significant stigma and social exclusion (Thomas, 2005; Dickinson et al., 2019). P\&O health care providers have a unique opportunity to challenge this by engaging patients, who may be lifelong users of their services, and working together in partnership. Translating this co-production approach, more commonly used in HICs and in technology development, into LRSs could give PWD the opportunity to act as role models, improve empowerment and work together with health care providers to shape services that meet their needs and improve their outcomes (Ellen et al., 2017). As well as service users, opportunities also exist, and are welcomed, for engagement with government. Key assets and ongoing development projects, alongside skills gaps identified by the stakeholders themselves, present unique opportunities to leverage and build on existing assets to maximise impact.

\section{Conclusion}

This paper presents a conceptual stakeholder-led framework for determining barriers and facilitators to translational global healthcare research in a country-specific context and demonstrated its application on an exemplar situation of Cambodian prosthetics service delivery. Subsequent research and development work in this area needs to carefully manage and negotiate influencing factors to ensure initiatives (whether research or wider national development work) are sustainable and successful. The reports which result from this approach can be used to ensure that interpretations of stakeholder perspectives are valid and allow stakeholders to leverage consolidated sector-wide intelligence to their advantage, mobilising local, regional, and national drivers and influencers to change. In many respects, these reports can harness the values of these respective groups and encourage championing of initiatives that are recognised as locally owned by stakeholders and reflecting their perspectives. Cambodia, with its long, complex historical context and rich, increasingly diverse P\&O services, could be used as a regional demonstration case, and it may be feasible that this country could pioneer innovative approaches for working systems for this LRS and capacity building. This level of engagement would not be possible without a value-embedded approach. 
Understanding the value perceived by various stakeholders is at the heart of this methodology. It is important to acknowledge that while this is not a research methodology, it provides important insights into the appropriate contexts from which meaningful qualitative and quantitative research can take place.

The methods presented provide a way to articulate the mission, vision, and goals of any proposed change and to effectively communicate these with stakeholders in a way that engages the personal and professional values that exist in their ecosystem. It provides a lens through which meaningful conversations can happen and context that can underpin the legacy of research and development.

\section{Acknowledgments}

This work was supported by the Engineering and Physical Sciences Research Council/ National Institute for Health Research Global Challenges Research Fund under grant EP/R014213/1 and the Royal Academy of Engineering under grant RAEng grant RF/130.

The authors thank Exceed Worldwide for facilitating, in particular Audrey Harte and Sam Simpson of the Exceed Research Network (ERN) for providing critical review. We also thank the University of Southampton's Institute for Life Sciences / FortisNet interdisciplinary musculoskeletal research network for supporting our preliminary work.

\section{Disclosure Statement}

We have no potential conflicts of, or competing interest relevant to this study.

\section{References}

AHRC (2016) AHRC Delivery Plan 2016-2020. Swindon, UK.

Bailey, S. (2015) ENSURING SUSTAINABILITY OF PHYSICAL REHABILITATION SERVICES IN CAMBODIA: ANALYSIS OF TRANSITION PROCESS.

Braun, V. and Clarke, V. (2013) Successful qualitative research: a practical guide for beginners. Los Angeles, USA: SAGE Publications Ltd.

CHAI/ATScale (2020) Product Narrative: Prostheses. London, UK. Available at: https://at2030.org/product-narrative-prostheses/.

Cobley, D. (2018) Disability and International Development. London, UK: Routeledge.

Dalal, A. K. (2010) 'Disability-Poverty Nexus: Psycho-Social Impediments to Participatory Development', Psychology and Developing Societies. SAGE Publications India, 22(2), pp. 409-437. doi: 10.1177/097133361002200208.

Dickinson, A. et al. (2019) Technologies to enhance quality and access to prosthetics and orthotics: the importance of a multidisciplinary, user-centred approach. WHO Global Report on Assistive Technology (GReAT) consultation. Geneva, Switzerland.

Dillon, M. P. et al. (2019) 'Prosthetics and Orthotics International welcomes qualitative research submissions', Prosthetics and Orthotics International. SAGE Publications Ltd STM, 43(4), pp. 366-368. doi: 10.1177/0309364619863922.

Ellen, M. E. et al. (2017) 'There Is Much to Learn When You Listen: Exploring Citizen Engagement in High- and Low-Income Countries.', World health \& population. Canada, 17(3), pp. 31-42. doi: 10.12927/whp.2017.25307. 
EW (2021) Exceed: equip, enable, empower.

Freeman, R. E. (1984) 'Stakeholder Management: Framework and philosophy', in Strategic Management: A Stakeholder Approach. Cambridge: Cambridge University Press, pp. 52-82.

Freeman, R. E., Harrison, J. S. and Zyglidopoulos, S. (2018) Stakeholder Theory. Cambridge: Cambridge University Press.

INVOLVE (2016) Public involvement in research and research ethics committee review. Available at: https://www.invo.org.uk/wp-content/uploads/2016/05/HRA-INVOLVE-updated-statement-2016.pdf.

Johnson, G., Scholes, K. and Whittington, R. (2008) 'Stakeholder mapping: the power/interest matrix', in Exploring Corporate Strategy. 8th edn. Essex, England: Pearson Education Limited, p. 156.

Jones, H. et al. (2021) 'Co-Creation Facilitates Translational Research on Upper Limb Prosthetics', Prosthesis . doi: 10.3390/prosthesis3020012.

Khan, F. et al. (2018) 'Capacity-building in clinical skills of rehabilitation workforce in low- and middleincome countries.', Journal of rehabilitation medicine. Sweden, 50(5), pp. 472-479. doi: 10.2340/16501977-2313.

Lewin, K. (1951) 'Field Theory of Social Science: Selected Theoretical Papers. (Edited by Dorwin Cartwright.) Pp. xx, 346. New York: Harper \& Brothers, 1951. \$5.00', The ANNALS of the American Academy of Political and Social Science. SAGE Publications Inc, 276(1), pp. 146-147. doi: 10.1177/000271625127600135.

Lindley, R. I. et al. (2017) 'Family-led rehabilitation after stroke in India (ATTEND): a randomised controlled trial', The Lancet. Elsevier, 390(10094), pp. 588-599. doi: 10.1016/S0140-6736(17)314472.

Marino, M. et al. (2015) 'Access to prosthetic devices in developing countries: Pathways and challenges', in 2015 IEEE Global Humanitarian Technology Conference (GHTC), pp. 45-51. doi: 10.1109/GHTC.2015.7343953.

Matter, R. et al. (2017) 'Assistive technology in resource-limited environments: a scoping review.', Disability and rehabilitation. Assistive technology. England, 12(2), pp. 105-114. doi: 10.1080/17483107.2016.1188170.

More, E., Probert, D. and Phaal, R. (2015) 'Improving long-term strategic planning: An analysis of STEEPLE factors identified in environmental scanning brainstorms', in 2015 Portland International Conference on Management of Engineering and Technology (PICMET), pp. 381-394. doi: 10.1109/PICMET.2015.7273126.

Mtika, W., Wilcox, H. and Feune De Colombi, N. (2021) Stakeholder Engagement for Applying Research to Policy and Practice for Health (ARCH). doi: https://doi.org/10.48060/tghn.6.

NIHR (2012) Briefing notes for researchers: public involvement in NHS, public health and social care research, INVOLVE. Available at: https://www.invo.org.uk/wpcontent/uploads/2014/11/9938_INVOLVE_Briefing_Notes_WEB.pdf (Accessed: 8 July 2020).

Osterwalder, A. et al. (2014) Value Proposition Design: How to create products and services customers want. Hoboken, New Jersey, USA: John Wiley \& Sons, Inc.

Porter, M. (1979) 'How competitive forces shape strategy', Harvard Business Review, 57(2), pp. 137145.

Saeedi, P. et al. (2019) 'Global and regional diabetes prevalence estimates for 2019 and projections for 2030 and 2045: Results from the International Diabetes Federation Diabetes Atlas, 9(th) edition.', Diabetes research and clinical practice. Ireland, 157, p. 107843. doi: 10.1016/j.diabres.2019.107843.

Sanders, E. B.-N. and Stappers, P. J. (2008) 'Co-creation and the new landscapes of design', CoDesign. 
Taylor \& Francis, 4(1), pp. 5-18. doi: 10.1080/15710880701875068.

Sexton, S. (2015) Rehabilitation of people with physical disabilities in developing countries. Køge, Denmark.

Thomas, P. (2005) Poverty reduction and development in Cambodia: Enabling disabled people to play a role. Available at:

https://assets.publishing.service.gov.uk/media/57a08c5840f0b652dd001280/PolicyProject_cambodia .pdf.

United Nations (2015) Transforming our world: the 2030 agenda for sustainable development. Available at: https://sustainabledevelopment.un.org/post2015/transformingourworld/publication.

Whittington, R. et al. (2020) 'Macro-environment analysis', in Exploring Strategy. 12th edn. Harlow, England: Pearson Education Limited, pp. 34-58.

WHO (2008) Task shifting: rational redistribution of tasks among health workforce teams: global recommendations and guidelines. Geneva.

WHO (2013) World Report in Disability. Geneva, Switzerland. doi: 10.1017/CBO9781107415324.004.

WHO (2017a) The need to scale up rehabilitation. Geneva.

WHO (2017b) 'WHO Standards for Prosthetics and Orthotics'.

WHO (2019) Rehabilitation in health systems: guide for action. Geneva.

Zhou, B. et al. (2019) 'Caregiver-Delivered Stroke Rehabilitation in Rural China', Stroke. Lippincott Williams \& WilkinsHagerstown, MD, 50(7), pp. 1825-1830. doi: 10.1161/STROKEAHA.118.021558. 